\title{
MACROSCOPIC MODELING AND CONTROL OF EMISSION IN URBAN ROAD TRAFFIC NETWORKS
}

\author{
Alfréd Csikós ${ }^{1}$, Tamás Tettamanti ${ }^{2}$, István Varga ${ }^{3}$ \\ ${ }^{1}$ Systems and Control Laboratory, Institute for Computer Sciences and Control, \\ Hungarian Academy of Sciences, Budapest, Hungary \\ ${ }^{2,3}$ Dept of Control for Transportation and Vehicle Systems, \\ Budapest University of Technology and Economics, Budapest, Hungary
}

Submitted 5 May 2014; resubmitted 26 September 2014; accepted 7 November 2014

\begin{abstract}
This work suggests a framework for modeling the traffic emissions in urban road traffic networks that are described by the Network Fundamental Diagram (NFD) concept. Traffic emission is formalized in finite spatiotemporal windows as a function of aggregated traffic variables, i.e. Total Travel Distances (TTDs) in the network and network average speed. The framework is extended for the size of an urban network during a signal cycle - the size of a window in which the network aggregated parameters are modeled in the NFD concept. Simulations have been carried out for model accuracy analysis, using the microscopic Versit+Micro model as reference. By applying the macroscopic emission model function and the traffic modeling relationships, the control objective for pollution reduction has also been formalized. Basically, multi-criteria control design has been introduced for two criteria: maximization of the TTD and minimization of traffic emissions within the network.
\end{abstract}

Keywords: urban road traffic; macroscopic traffic emission; perimeter control; modeling; network.

\section{Introduction}

In our days, the conscious transport planning and decision-making are more and more expected (Bokor 2011). As consequence, beside the clear financial goals, environmental and social aspects must be taken into account as well. Traffic demands may easily exceed infrastructure capacity of urban areas causing oversaturated traffic situation. Although traditional traffic-responsive control methods may perform efficiently, extreme traffic (when demands overspill the capacity) may result in long congested time periods. Therefore, the concept of the Protected Network (PN) has been highlighted recently as an efficient solution to prevent traffic jams in certain urban networks.

A PN usually represents a city center or a dense urban area that needs protection against insatiate demands during rush hours. The proper traffic load of a $\mathrm{PN}$ can be controlled in several ways, e.g. congestion charge (Szendrő 2011; Zhang et al. 2014), or gate control. Moreover, other innovative techniques have been presented to affront traffic jams, i.e. temporary use of reversible lanes within the network (Cao et al. 2014) or road infrastructure booking (Soltész et al. 2011).
The gate (perimeter) control seems to be the one of the most effective tool for a PN to its direct and dynamic impact on traffic flows allowing an optimal traffic flow through the network gates. The traffic control of a PN is often related to the theory of the urban fundamental diagram, which was first proposed by Godfrey (1969). The theory is called both Macroscopic Fundamental Diagram (MFD) and Network Fundamental Diagram (NFD). The NFD concept has been widely investigated during the past decades, e.g. Mahmassani et al. (1987), Daganzo (2007), Keyvan-Ekbatani et al. (2013, 2014).

The applicable control methodologies have also appeared by using the traffic lights along the perimeter of the PN as controllable gates. Daganzo (2007) introduced a control rule based on time dependent switching conditions. The work of De Jong et al. (2013) analyzed the effect of different signal strategies within the PN on the shape of the NFD, and proposed a control system, separating the control along the links at the boundary of the PN and inside the PN. Keyvan-Ekbatani et al. (2013) provided a thorough description of the NFD model, and designed a linear feedback regulator control (PID control) for the described model dynamics. 
The works mentioned above intend to optimize traffic performance, by increasing the Total Travel Distances (TTDs) in the network. In our paper, a multi-criteria approach is presented, i.e. design of a controller to optimize emissions of the PN besides traffic performance optimization. Efforts have been made for the modeling and control of urban traffic emissions. The papers of $\mathrm{Li}$ and Shimamoto (2012), Stevanovic et al. (2012), Gori et al. (2012) and Lin et al. (2013) have presented microscopic approaches for modeling vehicular emissions near intersections. However, neither of them exploits the concept of NFD, or follows a macroscopic approach.

In this work, a novel approach is suggested and analyzed for the modeling of pollutant emissions in urban road networks. Moreover, a control objective statement for pollution reduction is also introduced. In this concept, the emission needs to be modeled and controlled with an important condition: the emission modeling framework must use the measurements of the existing traffic system model (i.e. no further measurements can be required). The model is based on the macroscopic traffic emission framework, introduced in Csikós and Varga (2012) and Csikós et al. (2013). In that work, the emission of traffic flow is formalized as a bivariate function of time and space (i.e. a distributed parameter system) and as a function of the macroscopic traffic variables. The traffic system model, used in our work relies on the system model proposed by Keyvan-Ekbatani et al. (2013) with certain modifications: extension of system states by considering the queuing dynamics and assumptions on the actuation delays.

The paper is organized as follows. First, the mathematical model of the system is stated. Thereafter, macroscopic modeling approach of urban emissions is suggested. Case studies are also introduced in order to investigate the accuracy of the model. Finally, the potential control objective for pollution reduction is formalized.

\section{Urban Road Traffic Model}

For the model, the same assumptions are taken as in Keyvan-Ekbatani et al. (2013) with certain modifications.

\subsection{Model Equations}

The basic and most important rule that has to be satisfied by a traffic network is the conservation law. For the $\mathrm{PN}$, it can be formalized as follows:

$$
T_{s}\left(Q_{i n}(k)+Q_{d}(k)-Q_{o u t}(k)\right),
$$

where: $k$ denotes the discrete time step index; $T_{s}$ in unit $[\mathrm{h}]$ is the discrete sample time step, in our case the signal cycle time.

Practically, Eq. (1) depicts the state variation during the time interval $\left[k T_{s},(k+1) T_{s}\right]$. State variable is represented by $N_{P N}(k)$, the number of vehicles within the $\mathrm{PN}$, given in passenger car equivalent $[\mathrm{PCE}]$ (the different types of road vehicles can be expressed in the ratio of private car). $Q_{i n}(k)=\sum_{j=1}^{n_{i n}} q_{i n, j}(k)$ in unit [PCE/h] is the sum of inflow of vehicles to the PN, whereas $n_{\text {in }}$ denotes the number of controlled gates. $Q_{d}(k)=\sum_{j=1}^{n_{d}} q_{d, j}(k)$ is the sum of uncontrolled inflow, and $n_{d}$ denotes the number of uncontrolled gates. $Q_{\text {out }}(k)=\sum_{j=1}^{n_{\text {out }}} q_{\text {out }, j}(k)$ is the sum of outflow of vehicles from the PN, with $n_{\text {out }}$ denoting the number of exit gates.

The second basic law that is used for the model is the concept of urban NFD (Daganzo 2007; Gartner, Wagner 2008). The NFD of the PN describes the relationship between the total travel distance $\left(T T D_{P N}\right.$, in unit [PCE.km]) and the total time spent $\left(T T S_{P N}\right.$, in unit [PCE.h]) within the PN (during a discrete step). These traffic variables can be obtained by following the concept of Keyvan-Ekbatani et al. (2013), reformalized by using the results of Ashton (1966):

$$
\begin{aligned}
& \operatorname{TTS}_{P N}(k)=T_{s} \sum_{j=1}^{n_{\text {link }}} N_{j}(k) ; \\
& \operatorname{TTD}_{P N}(k)=T_{s} \sum_{j=1}^{n_{\text {link }}} q_{j}(k) L_{j},
\end{aligned}
$$

where: $n_{\text {link }}$ denotes the number of links in the PN; $q_{j}(k)$ and $L_{j}$ in unit $[\mathrm{km}]$ denote the traffic flow and length of link $j$ respectively.

A full information control is assumed, i.e. that all traffic variables are measured and the exact number of vehicles in the PN can be calculated based on the following equation:

$$
N_{P N}(k)=\sum_{j=1}^{n_{\text {link }}} \rho_{j}(k) L_{j},
$$

where: $\rho_{j}(k)$ in unit $[\mathrm{PCE} / \mathrm{km}]$ denotes the traffic density on link $j$.

By using Eq. (4) the knowledge of the operational NFD can be supposed.

The fundamental relationship can be stated as follows:

$$
\operatorname{TTD}_{P N}(k)=F\left(\operatorname{TTS}_{P N}(k)\right)+\varepsilon(k),
$$

where: $F(\cdot)$ denotes the nonlinear function of the complete operational NFD, fitted to historic measurements. $\varepsilon(k)$ denotes the fitting error, considered as noise in the system dynamics.

The network model also assumes that the total outflow $Q_{\text {out }}(k)$ of the $\mathrm{PN}$ is proportional to $\operatorname{TTD}_{P N}(k)$, satisfying the following equation:

$$
Q_{\text {out }}(k)=\Gamma \frac{T T D_{P N}(k)}{L T_{s}},
$$

where: $0 \leq \Gamma \leq 1$ is the network exit rate parameter; $L$ is the average link length in the PN. Coefficient $\Gamma$ can be fitted using the measurements of the total outflow of the network and $T T D_{P N}$. 
The actuator dynamics of traffic lights at controlled gate $j$ is described as follows:

$$
q_{i n, j}(k)=\beta_{j} q_{g, j}(k),
$$

where: $\beta_{j}$ is the portion of gated flow $\left(q_{g, j}\right)$ that enters the $\mathrm{PN},\left(0 \leq \beta_{j} \leq 1\right)$ and can be approximated by fitting to the measurements of $q_{i n, j}$ and $q_{g, j}$.

In addition to the model outlined in Keyvan-Ekbatani et al. (2013), our framework considers the queuing dynamics as well:

$$
l_{j}(k+1)=l_{j}(k)+T_{s}\left(q_{d e m, j}(k)-q_{i n, j}(k)\right),
$$

where: $l_{j}$ in unit $[\mathrm{PCE}]$ denotes the queue length at gate $j$ where the traffic demand $q_{d e m, j}$ emerges.

To sum up the differences between the proposed model and the one used in Keyvan-Ekbatani et al. (2013), for modeling simplifications, in our work a full information control system is supposed, i.e. the complete operational fundamental diagram is supposed to be known. Thus, for the estimation error $\varepsilon_{1}=0$ and for the operational fundamental diagram correction factors $A=1$ and $B=1$ are supposed. Apart from this, by placing the gates at the boundary of the $\mathrm{PN}$, the delay of the actuator system is eliminated and $\tau=0$ is supposed. The model, however, is extended by modeling the queues at the gates to optimize inflow allocation.

\subsection{State Space Model}

The dynamic model equations can be reformulated in a nonlinear state-space system framework as follows:

$$
\begin{aligned}
& \left(\begin{array}{c}
N_{P N}(k+1) \\
l_{1}(k+1) \\
\vdots \\
l_{n_{\text {in }}}(k+1)
\end{array}\right)=\left(\begin{array}{c}
N_{P N}(k)-T_{s} \frac{\Gamma}{L} F\left(N_{P N}(k)\right) \\
l_{1}(k) \\
\vdots \\
l_{n_{i n}}(k)
\end{array}\right)+ \\
& \left(\begin{array}{c}
n_{s} \sum_{j=1}^{n} q_{i n, j}(k) \\
-T_{s} q_{i n, 1}(k) \\
\vdots \\
-T_{s} q_{i n, n_{i n}}(k)
\end{array}\right)+\left(\begin{array}{c}
T_{s} \sum_{j=1}^{n_{d}} \bar{q}_{d, j}(k) \\
T_{s} q_{d e m, n_{1}}(k) \\
\vdots \\
T_{s} q_{d e m, n_{i n}}(k)
\end{array}\right) .
\end{aligned}
$$

In Eq. (9), the second and third terms include the effect of control inputs and disturbances, respectively.

\subsection{System Variables}

As state variables, the vehicle number in the PN $N_{P N}$ and queue lengths of the controlled gates $l_{1}, \ldots, l_{n_{\text {in }}}$ are considered as state variables:

$$
x(k)=\left[N_{P N}(k), l_{1}(k), \ldots, l_{n_{\text {in }}}(k)\right]^{T} \in \mathfrak{R}^{n_{\text {in }}+1} .
$$

Disturbances are collected in the following vector:

$$
\begin{aligned}
& d(k)=\left[\bar{q}_{d, 1}(k), \ldots, \bar{q}_{d, n_{d}}(k), q_{d e m, 1}(k), \ldots,\right. \\
& \left.q_{d e m, n_{i n}}(k)\right]^{T} \in \Re^{n_{d}}+n_{i n},
\end{aligned}
$$

where: $\bar{q}_{d, j}\left(j=1, \ldots, n_{d}\right)$ is the nominal flow through uncontrolled gate $j$ and $q_{d e m, n_{j}}\left(i=1, \ldots, n_{\text {in }}\right)$ denotes the traffic demand emerging at controlled gate $i$.

The input vector is in the form:

$$
u(k)=\left[q_{i n, 1}(k), \ldots, q_{i n, n_{i n}}(k)\right]^{T} \in \Re^{n_{i n}},
$$

where: $q_{i n, j}\left(j=1, \ldots, n_{i n}\right)$ is the gated flow via gate $j$.

\subsection{Case Study}

The proposed model is applied for a model network (Fig. 1) in a microscopic traffic simulator. The test network is located in the 6th district of Budapest, and all its streets are one-way streets with no signalized intersections inside. Link lengths are similar with an average of $0.143 \mathrm{~km}$. Along with the topology, the loads at the gates are considered homogeneous. The network can be entered via six controlled and six uncontrolled gates, and escaped via nine exit gates as depicted in Fig. 1. As a result of the above considerations, homogeneous traffic conditions are present in the model network, and the concept of NFD modeling can be adopted for the case study network.

Firstly, the NFD has been identified for the model network. For this end, simulations were run with different traffic demands, representing the low demands and rush hours as well. The result of the eight different, one-hour-long simulations and the fitted fundamental function $F(\cdot)$ of Eq. (5) are plotted in Fig. 2.

The best fit is obtained by a 4 th-order polynomial function with appropriate parameters $A, B, C, D, E$ :

$$
F(T T S)=A \cdot T T S^{4}+B \cdot T T S^{3}+C \cdot T T S^{2}+D \cdot T T S+E .
$$

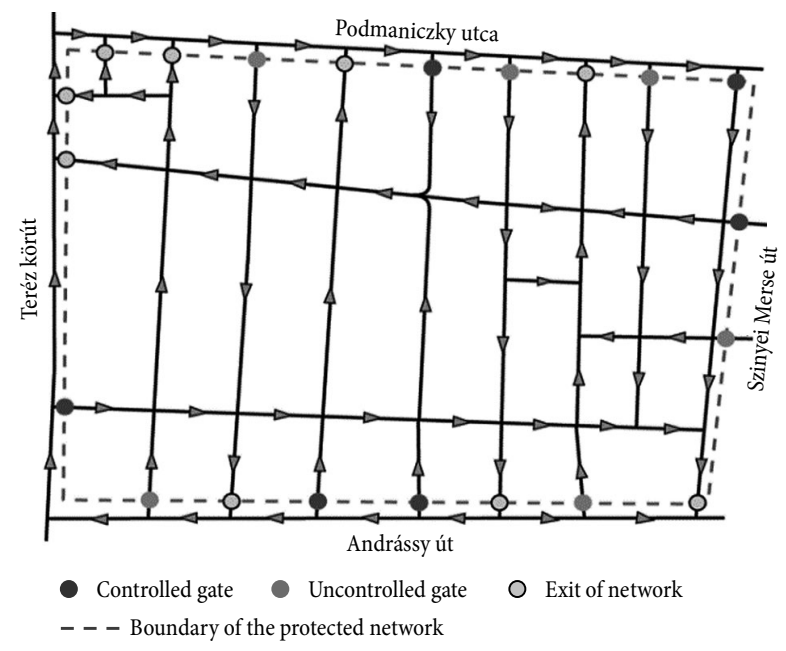

Fig. 1. Network layout 


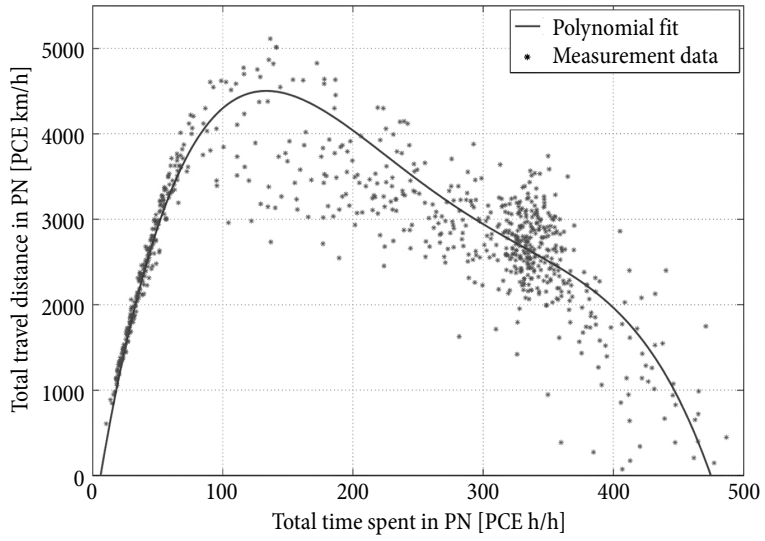

Fig. 2. NFD Fundamental diagram of the PN

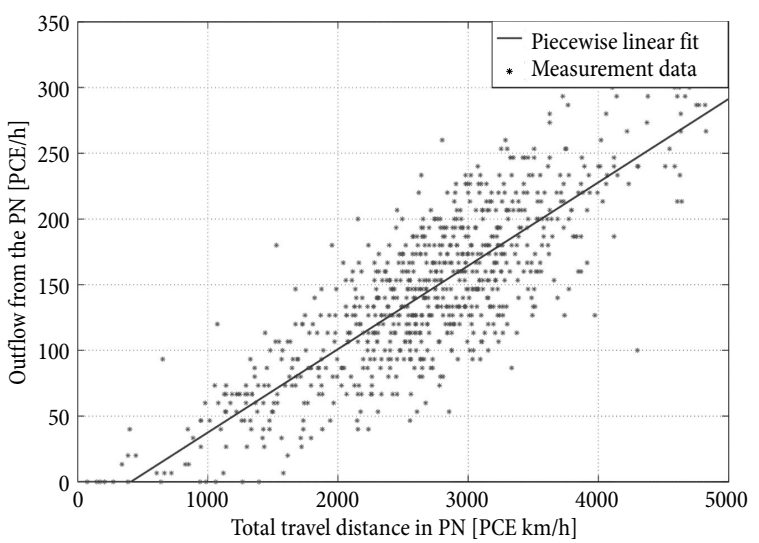

Fig. 3. Linear regression for a parameter $\Gamma$

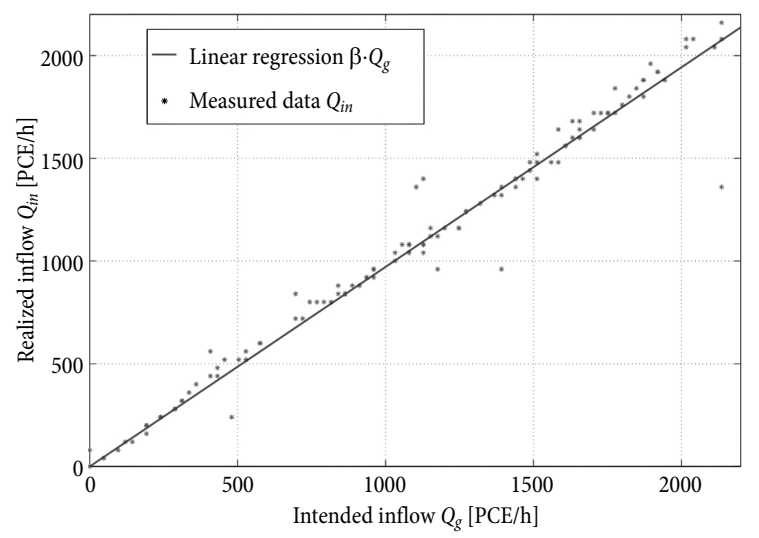

Fig. 4. Linear regression for parameter $\beta$

The second step is the identification of a model parameter $\Gamma$ based on the relationship in Eq. (6). This is carried out by a linear regression between the measurement data of $Q_{\text {out }}$ and $T T S_{P N}$. The linear regression results in the following formula is illustrated by Fig. 3:

$\Gamma= \begin{cases}0, & \text { if } T T D_{P N} \leq 45[\mathrm{PCE} \cdot \mathrm{km} / \mathrm{h}] ; \\ 0.063 \cdot T T D_{P N}-25.8, & \text { otherwise. }\end{cases}$
The third parameter to be identified is $\beta_{j}$ of Eq. (7), which describes the relationship between the intended and realized inflow through a gate. In our approach, an average parameter $\beta$ is considered representing $\beta_{j}$ for each $j$ gate. An appropriate linear regression has been carried out based on the measurement data as shown in Fig. 4.

\section{Macroscopic Modeling of Emission in Urban Traffic Networks}

The modeling of the vehicular emissions of a PN follows the same approach as used in the traffic modeling framework: the overall emissions of the $\mathrm{PN}$ is expressed using aggregated traffic variables: Total Travel Distance (TTD) and Total Time Spent (TTS).

The use of aggregated traffic variables for flow emission modeling has already been introduced in the paper of Csikós and Varga (2012). In that work, similarly to the traffic flow variables in the continuum models, emission of traffic is also considered as a function over space and time (i.e. as a distributed parameter system variable). Analogously to traffic performances (such as TTD and TTS), flow emission can be expressed as a function of macroscopic traffic variables in infinitesimal spatiotemporal rectangles using the following approach.

Consider a homogeneous traffic moving along a road and analyze the traffic variables on the short road segment $\left[l_{0} ; l_{0}+L\right]$ for a short period of time $\left[t_{0} ; t_{0}+T\right]$ (analysis in a spatiotemporal window of size $L \times T)$. The traffic density in an infinitesimal spatiotemporal window $L \times T$ is equal to the TTS in that window (Ashton 1966):

$$
\rho\left[l_{0} ; l_{0}+L\right] \times\left[t_{0} ; t_{0}+T\right]=\rho_{L \times T}=\frac{T T S_{L \times T}}{L \cdot T},
$$

where: $\rho_{L \times T}$ denotes the traffic density in $L \times T$. In a similar manner, average traffic flow in the spatiotemporal window is equal to the TTD in that window (Ashton 1966):

$$
q_{\left[l_{0} ; l_{0}+L\right] \times\left[t_{0} ; t_{0}+T\right]}=q_{L \times T}=\frac{T T D_{L \times T}}{L \cdot T},
$$

where: $q_{L \times T}$ denotes the traffic flow measured in $L \times T$. This approach is extended to emission modeling, ordered to the following subsections.

\subsection{Emission of a Single Vehicle}

The emission of a single vehicle is described by the emission factor function ef in unit $[\mathrm{g} / \mathrm{km}]$ (i.e. the distance specific emission) as a function of vehicle speed $v$. E.g. emission factor of pollutant $\mathrm{CO}$ can be considered with the following formula by using the model Copert IV (Ntziachristos et al. 2000; Franco et al. 2013):

$$
e f_{C O}=\frac{\beta_{m}^{C O} v^{m}+\ldots+\beta_{1}^{C O} v+1}{\alpha_{n}^{C O} v^{n}+\ldots+\alpha_{1}^{C O} v+1},
$$

i.e. as a rational fractional function, in which parameters $\beta_{m}^{C O}, \ldots, \beta_{1}^{C O}$ and $\alpha_{n}^{C O}, \ldots, \alpha_{1}^{C O}$ are determined by curve fitting to vehicle dynamometer measurements of prespecified driving cycles. 


\subsection{Overall Emission of the Traffic Flow}

The overall emission of the traffic flow for pollutant $p$ can be formalized within the spatiotemporal rectangle $L \times T$ in unit $[\mathrm{g}]$ as follows:

$$
E_{L \times T}^{p}=e f P\left(v_{L \times T}\right) \cdot T T D_{L \times T} .
$$

On a single road segment, substituting the generalized definition (16) to (18):

$$
E_{L \times T}^{p}=e^{p}\left(v_{L \times T}\right) \cdot q_{L \times T} \cdot L \cdot T .
$$

Thus, the total produced emission of traffic can be expressed as a function of traffic flow and traffic mean speed for a spatiotemporal rectangle $L \times T$.

Remark: The model so far is simply applicable for lumped motorway systems, in a spatiotemporally discrete framework where the default unit is a motorway segment of length $L$ during a sample time step $T$ of length.

\subsection{Emission of the PN}

In the following, the notation $L_{P N}$ represents the links within the PN:

$$
L_{P N}=\left\{l_{i}\right\}, i=1, \ldots, n_{\text {link }} .
$$

The total emission in the PN is the sum of the emissions of the network links:

$$
E_{L_{P N} \times T}^{p}=\sum_{i=1}^{n_{\text {link }}} E_{l_{i} \times T}^{p} .
$$

However, the emission of the PN needs to be calculated not by links, but for the whole network, using the aggregated variables $T T S_{P N}$ and $T T D_{P N}$. Although the available measurements include the link-wise traffic flows, from which $T T D_{P N}$ is calculated (see Eq. (3)), and the number of vehicles within the network is available from Eq. (4), average speeds are not supposed to be measured for each link $i$ in our work. Nevertheless, average cruising speed in unit $[\mathrm{km} / \mathrm{h}]$ of the network can be expressed using the basic relationship among the traffic variables (Ashton 1966):

$$
v_{L_{P N} \times T}=\frac{q_{L_{P N} \times T}}{\rho_{L_{P N} \times T}} .
$$

Substituting Eqs (15 and 16) to Eq. (22):

$$
v_{L_{P N} \times T}=\frac{T T D_{L_{P N} \times T}}{T T S_{L_{P N} \times T}} .
$$

The average cruising speed $v_{P N}$ is supposed to represent the speed conditions of the $\mathrm{PN}$ in the spatiotemporal rectangle $L_{P N} \times T$, and is substituted to the framework.

\subsection{Emission of the PN via Aggregated Network Parameters}

The emission of pollutant $p$, emerging in the $\mathrm{PN}$ during time $T$ can be stated as follows, using Eqs $(23,18)$ :

$$
E_{L_{P N} \times T}^{p}=e f p\left(v_{L_{P N} \times T}\right) \cdot T T D_{L_{P N} \times T}
$$

where: $v_{L_{P N} \times T}$ is calculated as in Eq. (23). Emission of pollutant $p$ in the $\mathrm{PN}$, in spatiotemporally discrete form:

$$
E_{P N}^{p}(k)=e^{p}\left(v_{P N}(k)\right) \cdot T T D_{P N}(k),
$$

where:

$$
v_{P N}(k)=\frac{T T D_{P N}(k)}{T T S_{P N}(k)} .
$$

By using the above formulae, the emission of the PN can be stated using aggregated network parameters $v_{P N}(k)$ and $T T D_{P N}(k)$. However, it needs to be analyzed, how the extension of infinitesimal spatiotemporal increments $L$ and $T$ effects the accuracy of emission calculation. For this end, simulations are run in which the microscopic emission of the vehicles, and link-wise emissions of the traffic Eq. (21) are simulated. The calculated emissions (using Eq. (25)) are compared to the link-wise calculations and the reference, the microscopic emissions.

Remark: the emission of the vehicles stuck outside is considered with zero emissions. This consideration can be justified by the assumption of the presence of start-stop engine systems which is a wide spread accessory of modern vehicles. Moreover, this action can also be suggested by fixed message signs at the gates.

\section{Analysis of Model Accuracy Based on Simulations}

The accuracy of the developed model framework is analyzed through simulations. The suggested model framework, i.e. the modeling of the emission of the PN using aggregated traffic variables, is utilized with the Copert IV average speed model. This emission calculation is compared to two levels of emission modeling:

1. The emission as a sum of each link's emission, calculated by macroscopic measurements of the links (link-wise emission modeling), also using the Copert IV average speed modeling for link emissions.

2. The microscopic description which is considered reference, using the Versit+Micro model (Smit et al. 2007) via the EnViVer add-on module of Vissim.

Two scenarios are used for the comparison. The first scenario represents a rush hour situation with changing traffic loads and a fixed time signal control. The second scenario realizes the same traffic load, (i.e. a rush hour traffic with changing loads), but uses a PID controller with oscillations. The oscillations provide an opportunity to analyze the model accuracy for different state values.

\subsection{Simulation Environment}

A microscopic traffic simulator: Vissim was used alongside its offline microscopic emission calculation add-on, EnViVer. EnViVer is based on the microscopic emission model Versit+Micro (Smit et al. 2007). Vissim enables 
signal controller commanding and loop-detector measurements through the COM interface by external programs like Matlab. The properties of the used framework are detailed in Tettamanti and Varga (2012).

For the simulations, the following parameters are set. The sampling time is chosen as the signal controller cycle time: $T_{s}=90 \mathrm{~s}$. The simulations run for $7200 \mathrm{~s}$. The emission factor function of the simulation is as follows (considering the following vehicle type: passenger car with a Euro 5 gasoline engine, under $1.4 \mathrm{~L}$ engine displacement):

$$
e f(v)=\frac{\alpha+\gamma v+\varepsilon v^{2}}{1+\beta v+\delta v^{2}}
$$

with the following parameters: $\alpha=0.5247 ; \beta=0$; $\gamma=-0.01 ; \delta=0 ; \varepsilon=9.36 \cdot 10^{-5}$.

\subsection{Simulation Results - Scenario 1}

The accuracy of the model can be best examined, if a wide range of the state domain is used within a simulation. For this end, two scenarios are modeled. First, a congested situation is presented by Scenario 1 .

The gate control and disturbance signals of Scenario 1 are plotted in Fig. 5, whereas the network performances ( $T T D$, network average speed) are presented in Fig. 6.
This basic scenario simulates congesting conditions, thus mainly low speeds are present. Fig. 7 highlights the model accuracy. The emission using aggregated variables (and network average speed) is very similar to the link-wise emission calculation. However, both have higher variations than the real emissions, calculated by Versit+Micro. The reason for this is the high sampling time (equal to the cycle time). By reducing the sampling time, the variation can be reduced. The relative error statistics of Scenario 1 are summarized in Table 1.

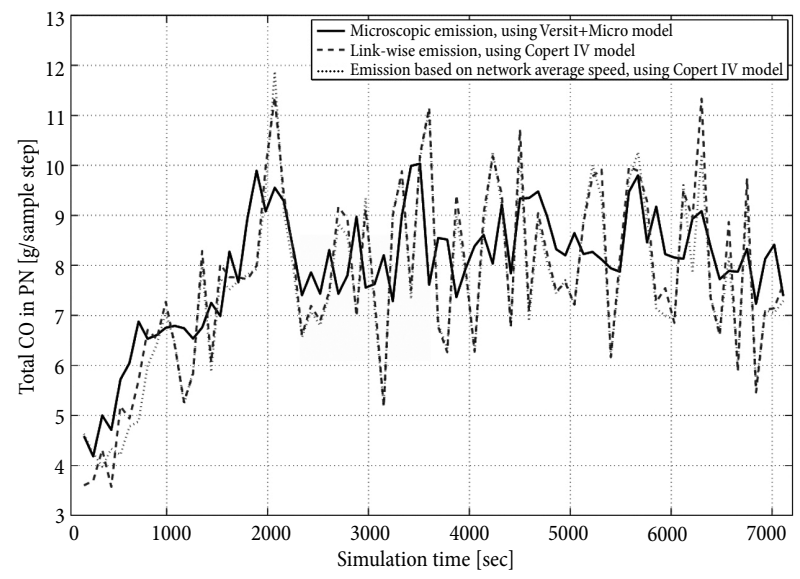

Fig. 7. Scenario 1: network emissions
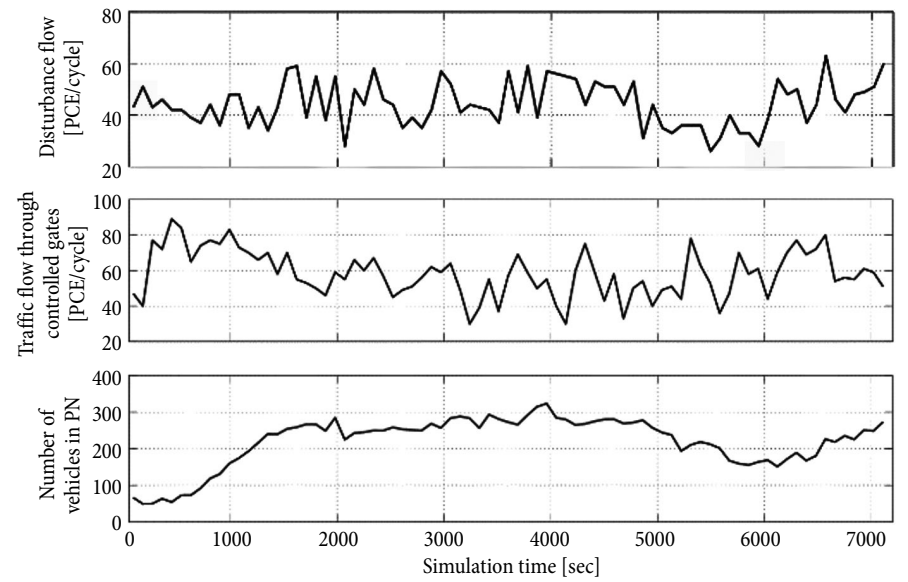

Fig. 5. Scenario 1: gate inputs and number of vehicles in PN

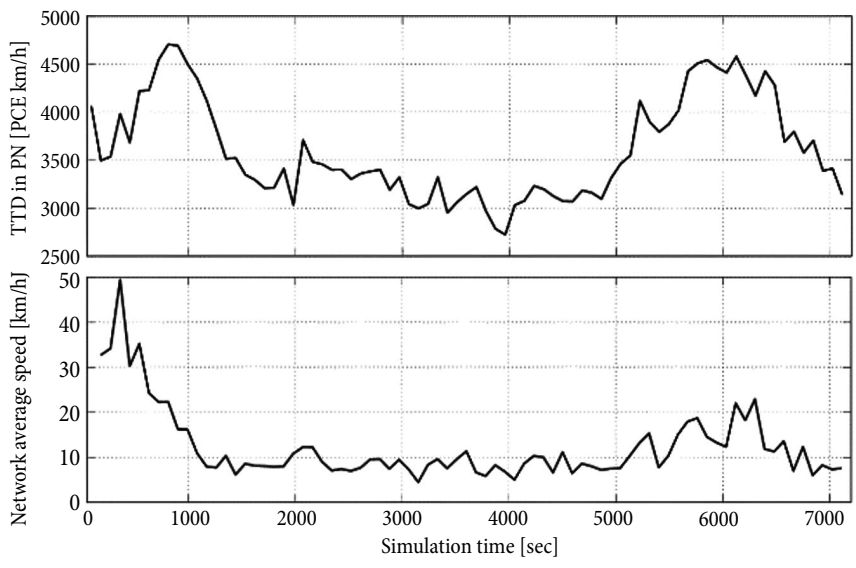

Fig. 6. Scenario 1: network performances 
Table 1. Relative error results of Scenario 1 compared to the microscopic model

\begin{tabular}{lcc}
\hline & $\begin{array}{l}\text { Link-wise } \\
\text { emission }\end{array}$ & $\begin{array}{c}\text { Emission based on } \\
\text { aggregated variables }\end{array}$ \\
\hline $\begin{array}{l}\text { Average relative } \\
\text { error }\end{array}$ & $16.7 \%$ & $18.2 \%$ \\
\hline
\end{tabular}

The relative errors of the Scenario 1 are high relative to the reference emission produced by the microscopic model. Nevertheless, the link-wise emissions and the emission values based on network-average traffic variables are very similar with small $(<2 \%$ relative errors). Thus, the network average emission model shows good accuracy in Scenario 1.

\subsection{Simulation Results - Scenario 2}

Scenario 2 features a PID controller, which is capable of preventing the congestion, however, with oscillations. Thus, a wide range of the domain is covered by the state dynamics and accuracy can be analyzed in case of extreme state values.

The gate control and disturbance signals of Scenario 2 are plotted in Fig. 8, whereas the network performances (TTD, network average speed) are presented in Fig. 9.
In this scenario both high and low traffic accumulation is present, thus the accuracy can be analyzed through both high and low speeds. Fig. 10 also highlights the model accuracy. In this case, the emission using aggregated variables shows less resemblance to the linkwise emission calculation. Again, higher variations can be present in both macroscopic emissions relative to the real emissions, calculated by Versit+Micro. The relative error statistics of Scenario 2 are tabulated into Table 2.

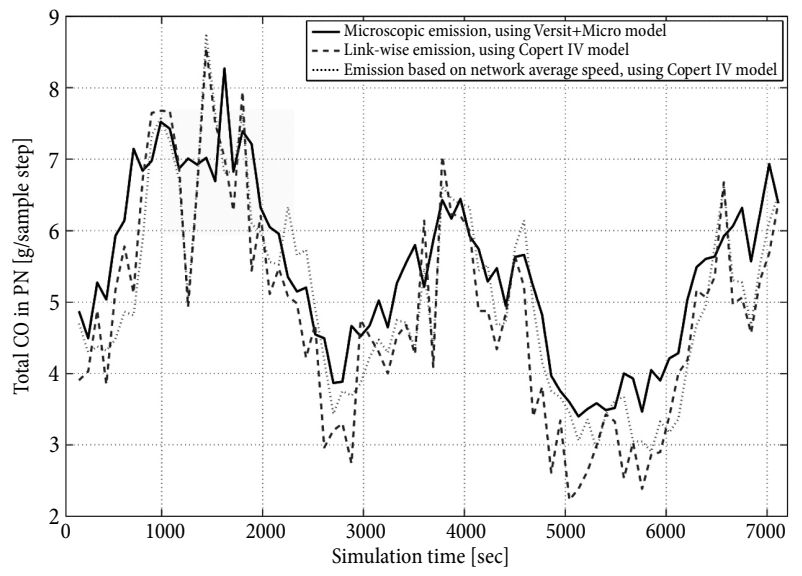

Fig. 10. Scenario 2: network emissions
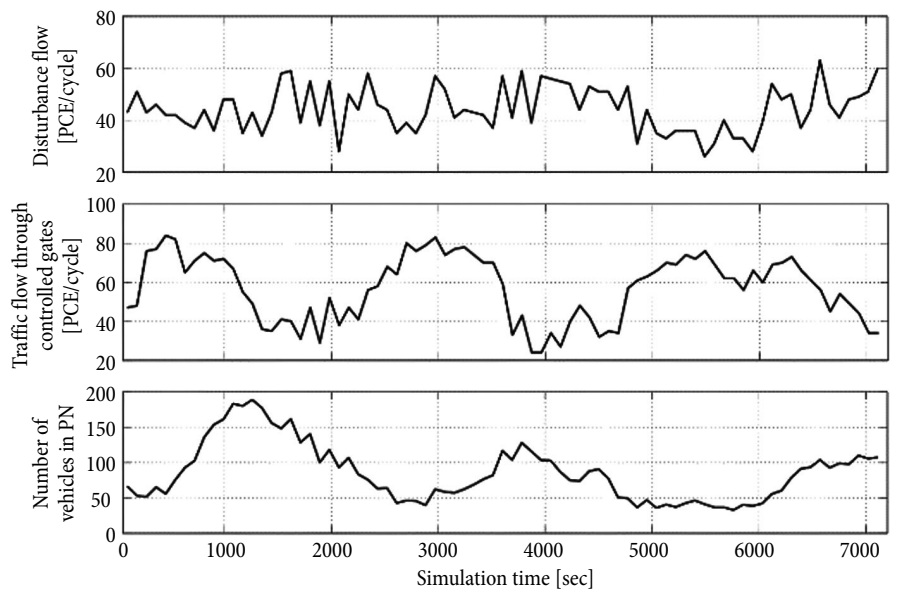

Fig. 8. Scenario 2: gate inputs and number of vehicles in PN

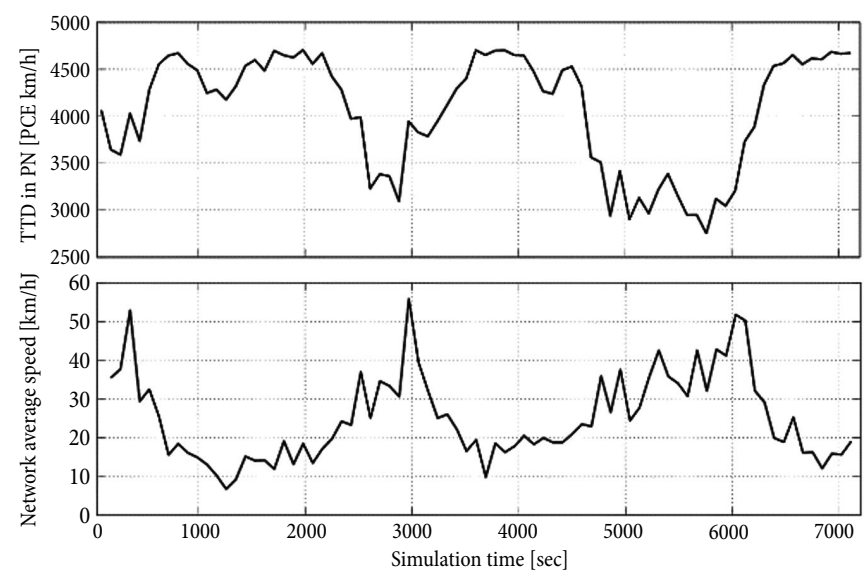

Fig. 9. Scenario 2: network performances 
Table 2. Relative error results of Scenario 2 compared to the microscopic model

\begin{tabular}{lcc}
\hline & $\begin{array}{c}\text { Link-wise } \\
\text { emission }\end{array}$ & $\begin{array}{c}\text { Emission based on } \\
\text { aggregated variables }\end{array}$ \\
\hline $\begin{array}{l}\text { Average relative } \\
\text { error }\end{array}$ & $17.2 \%$ & $19.7 \%$ \\
\hline
\end{tabular}

According to Scenario 2 link-wise emissions and the emission values based on network-average traffic variables are very similar again, with small $(<2 \%$ relative errors). Thus, the network average emission model shows good accuracy in Scenario 2 as well.

\section{Control Objective Statement}

For a future multi-criteria control, design is carried out for two criteria. The first control objective is the optimization of the traffic performance: maximizing the total travel distance within the $\mathrm{PN}$, while minimizing the queues at perimeter gates. The second control objective is the minimization of traffic emissions within the PN. The objectives for the control criteria are stated separately, and turned to regulator-type control objective functions. By using the recast formulae, an overall cost function is composed as a weighted sum of the criteria. In this section, the control objective statement for emission optimization within the PN is stated.

The control goal is the minimization of network emissions in each step:

$$
\begin{aligned}
& J_{e m}=\sum_{k=1}^{K}\left\|E_{P N}(k)\right\|_{2}^{2}= \\
& \sum_{k=1}^{K}\left\|e f\left(v_{P N}(k)\right) \operatorname{TTD}_{P N}(k)\right\|_{2}^{2} .
\end{aligned}
$$

This cost function needs to be recast in the following form, to get a regulator problem:

$$
J_{e m}=\sum_{k=1}^{K}\left\|E_{P N}\left(N_{P N}\right)(k)\right\|_{2}^{2},
$$

i.e. as a function of the state variable: the number of vehicles in PN (or equally, the TTS in the PN). By using the model functions Eqs (5 and 2), the function of $T T D_{P N}=f\left(N_{P N}\right)$ in Eq. (28) is already stated. Furthermore, the composition of functions ef $\left(v_{P N}\left(N_{P N}\right)\right)$ also needs to be formalized.

First, $e f(v)$ is analyzed. Eq. (17) presents the emission factor function, which is a monotonously decreasing function in the speed domain of urban driving cycles (Gois et al. 2007). Fig. 11 illustrates the example of an emission factor function.

Second, $v\left(N_{P N}\right)$ is analyzed. The relationship is often used in traffic modeling, as the equilibrium speeddensity function of the first order macroscopic traffic description. In case of an urban network, the interpretation of the phenomenon 'equilibrium speed' is not straightforward. Nevertheless, analogously to the NFD diagram, a relationship can be observed between the

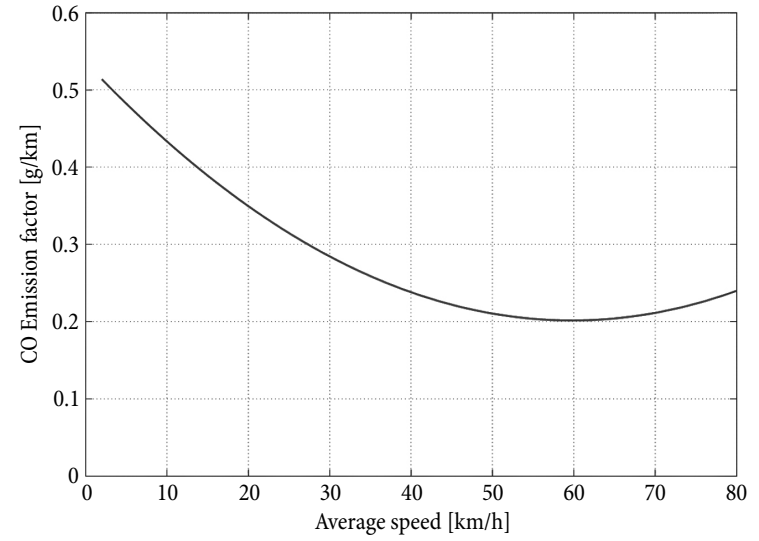

Fig. 11. Emission factor function of CO pollution (passenger car equipped with a Euro 5 gasoline engine, under $1.4 \mathrm{~L}$ )

network average speed and the number of vehicles in the network.

For illustration, similarly to the NFD diagram, a fourth-order polynomial fitting was applied for the case study dataset. The network average speed is modeled as a function of TTS (or equally, the number of vehicles within the network), by the following formula:

$v(T T S)=a \cdot T T S^{4}+b \cdot T T S^{3}+c \cdot T T S^{2}+d \cdot T T S+e$,

with appropriate parameters $a, b, c, d, e$.

The measurement data and the polynomial fit of Eq. (30) are plotted in Fig. 12.

Substituting the network average speed function Eq. (30) to Eq. (4), the composition of functions ef $\left(v_{P N}\left(N_{P N}\right)\right)$ can be stated in explicit form.

By using Eqs (30, 4 and 5), the objective function Eq. (28) can be formalized as a function of the state variable $N_{P N}$ :

$J_{e m}=\sum_{k=1}^{K}\left\|e f\left(v_{P N}\left(N_{P N}(k)\right)\right) \operatorname{TTD}_{P N}\left(N_{P N}(k)\right)\right\|_{2}^{2}$.

The function stated in Eq. (31) alongside with emission data of simulations is plotted in Fig. 13. The function has a minimum at $N_{P N}=0$, which is used as the regulator set point.

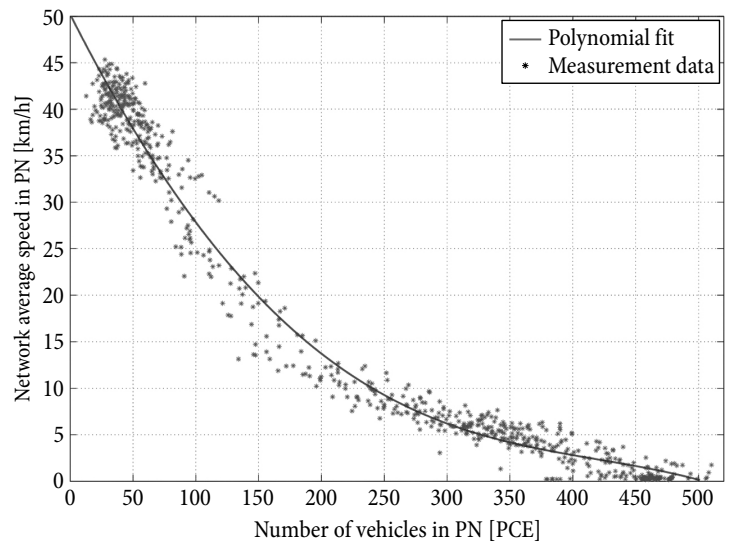

Fig. 12. Network average speed function 


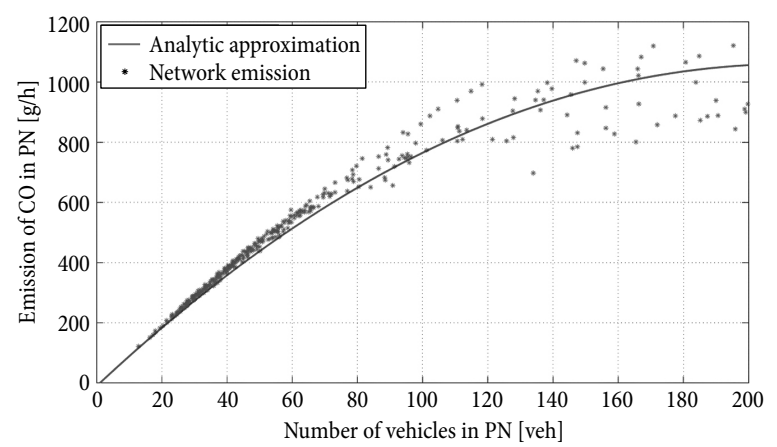

Fig. 13. Network emission as a function of number of vehicles

By itself, cost function Eq. (31) is not applicable for a control system as the optimal performance could be reached by keeping the traffic outside the PN. However, in a multi-criteria control design (which is a part of future research directions), it can be featured as a control criterion.

\section{Conclusions}

A macroscopic framework has been suggested for emission modeling in urban road traffic networks that are described by the concept of Network Fundamental Diagram (NFD). It has been shown that emission can be formalized in finite spatiotemporal windows as a function of Total Travel Distances (TTDs) and network average speed within the network.

Two case studies have also been carried out to examine the accuracy of the model. The simulation results confirmed the applicability of the concept. Compared to the relatively accurate microscopic models, the relative errors of the proposed macroscopic approach remained under $20 \%$ at all times. This is a reasonable compromise considering the unrealistic measurement demand in case of microscopic modeling.

\section{Acknowledgements}

This work is connected to the scientific program of EITKIC-12-1-2012-0001 project (supported by the Hungarian Government, managed by the National Development Agency, financed by the Research and Technology Innovation Fund), and TÁMOP-4.2.2.C-11/1/KONV2012-0012: Smarter Transport project (supported by the Hungarian Government, co-financed by the European Social Fund).

\section{References}

Ashton, W. D. 1966. The Theory of Traffic Flow. London: Methuen.

Bokor, Z. 2011. Calculation model for transport costing, Periodica Polytechnica - Transportation Engineering 39(1): 43-47. http://dx.doi.org/10.3311/pp.tr.2011-1.08

Cao, Y.; Zuo, Z.; Xu, H. 2014. Analysis of traffic conflict characteristic at temporary reversible lane, Periodica Polytechnica - Transportation Engineering 42(1): 73-76.

http://dx.doi.org/10.3311/PPtr.7068
Csikós, A.; Varga, I. 2012. Real-time modeling and control objective analysis of motorway emissions, Procedia - Social and Behavioral Sciences 54: 1027-1036.

http://dx.doi.org/10.1016/j.sbspro.2012.09.818

Csikós, A.; Varga, I.; Hangos, K. M. 2013. A simple dynamic model for the dispersion of motorway traffic emission, in 16th International IEEE Conference on Intelligent Transportation Systems (ITSC), 6-9 October 2013, Hague, Netherlands, 1559-1564.

http://dx.doi.org/10.1109/ITSC.2013.6728452

Daganzo, C. F. 2007. Urban gridlock: macroscopic modeling and mitigation approaches, Transportation Research Part B: Methodological 41(1): 49-62. http://dx.doi.org/10.1016/j.trb.2006.03.001

De Jong, D.; Knoop, V. L.; Hoogendoorn, S. P. 2013. The effect of signal settings on the macroscopic fundamental diagram and its applicability in traffic signal driven perimeter control strategies, in 16th International IEEE Conference on Intelligent Transportation Systems (ITSC), 6-9 October 2013, Hague, Netherlands, 1010-1015.

http://dx.doi.org/10.1109/ITSC.2013.6728364

Franco, V.; Kousoulidou, M.; Muntean, M.; Ntziachristos, L.; Hausberger, S.; Dilara, P. 2013. Road vehicle emission factors development: a review, Atmospheric Environment 70: 84-97. http://dx.doi.org/10.1016/j.atmosenv.2013.01.006

Gartner, N. H.; Wagner, P. 2008. Analysis of traffic flow characteristics on signalized arterials, Transportation Research Record 1883: 94-100. http://dx.doi.org/10.3141/1883-11

Godfrey, J. W. 1969. The mechanism of a road network, Traffic Engineering and Control 11(7): 323-327.

Gois, V.; Maciel, H.; Nogueira, L.; Almeida, C.; Torres, P.; Mesquita, S.; Ferreira, F. 2007. A detailed urban road traffic emissions inventory model using aerial photography and GPS survey, in 16th Annual International Emission Inventory Conference - Emission Inventories: "Integration, Analysis, and Communications", 14-17 May 2007, Raleigh, North Carolina, 1-20. Available from Internet: http://www.epa. gov/ttn/chief/conference/ei16/session9/gois.pdf

Gori, S.; La Spada, S.; Mannini, L.; Nigro, M. 2012. Within-day dynamic estimation of pollutant emissions: a procedure for wide urban network, Procedia - Social and Behavioral Sciences 54: 312-322. http://dx.doi.org/10.1016/j.sbspro.2012.09.750

Keyvan-Ekbatani, M.; Papageorgiou, M.; Papamichail, I. 2013. Urban congestion gating control based on reduced operational network fundamental diagrams, Transportation Research Part C: Emerging Technologies 33: 74-87. http://dx.doi.org/10.1016/j.trc.2013.04.010

Keyvan-Ekbatani, M.; Papageorgiou, M.; Papamichail, I. 2014. Perimeter traffic control via remote feedback gating, Procedia - Social and Behavioral Sciences 111: 645-653. http://dx.doi.org/10.1016/j.sbspro.2014.01.098

Li, C.; Shimamoto, S. 2012. An open traffic light control model for reducing vehicles' $\mathrm{CO}_{2}$ emissions based on ETC vehicles, IEEE Transactions on Vehicular Technology 61(1): 97-110. http://dx.doi.org/10.1109/TVT.2011.2168836

Lin, S.; De Schutter, B.; Xi, Y.; Hellendoorn, H. 2013. Integrated urban traffic control for the reduction of travel delays and emissions, IEEE Transactions on Intelligent Transportation Systems 14(4): 1609-1619. http://dx.doi.org/10.1109/TITS.2013.2263843

Mahmassani, H.; Williams, J.; Herman, R. 1987. Performance of urban traffic networks, in Proceedings of the 10th International Symposium on Transportation and Traffic Theory, 1987, Amsterdam, Netherlands, 1-20. 
Ntziachristos, L.; Samaras, Z.; Eggleston, S.; Gorißen, N.; Hassel, D.; Hickman, A.-J.; Joumard, R.; Rijkeboer, R.; White, L.; Zierock, K.-H. 2000. COPERT III Computer Programme to Calculate Emissions from Road Transport: Methodology and Emission Factors (Version 2.1). Technical Report No 49. European Environment Agency. 86 p. Available from Internet: http://www.eea.europa.eu/publications/ Technical_report_No_49

Smit, R.; Smokers, R.; Rabé, E. 2007. A new modelling approach for road traffic emissions: VERSIT+, Transportation Research Part D: Transport and Environment 12(6): 414-422. http://dx.doi.org/10.1016/j.trd.2007.05.001

Stevanovic, A.; Stevanovic, J.; Kergaye, C. 2012. Environmental benefits of adaptive traffic control system: assessment of fuel consumption and vehicular emissions, in TRB 91st Annual Meeting Compendium of Papers DVD, 22-26 January 2012, Washington, DC, 1-15.

Soltész, T.; Kózel, M.; Csiszár, C.; Centgráf, T.; Benyó, B. 2011. Information system for road infrastructure booking, $\mathrm{Pe}$ riodica Polytechnica - Transportation Engineering 39(2): 55-62. http://dx.doi.org/10.3311/pp.tr.2011-2.02

Szendrö, G. 2011. Congestion charging in Budapest - a comparison with existing systems, Periodica Polytechnica Transportation Engineering 39(2): 99-103. http://dx.doi.org/10.3311/pp.tr.2011-2.09

Tettamanti, T.; Varga, I. 2012. Development of road traffic control by using integrated VISSIM-MATLAB simulation environment, Periodica Polytechnica - Civil Engineering 56(1): 43-49. http://dx.doi.org/10.3311/pp.ci.2012-1.05

Zhang, L.; Liu, H.; Sun, D. 2014. Comparison and optimization of cordon and area pricings for managing travel demand, Transport 29(3): 248-259.

http://dx.doi.org/10.3846/16484142.2014.914970 